\title{
Equivalent Stiffness Evaluations of Clamped Plates in Bolted Joints under Loading*
}

\author{
Tomohiro NARUSE** and Yoji SHIBUTANI*** \\ ${ }^{* *}$ Hitachi, Ltd. Mechanical Engineering Research Laboratory, \\ 832-2, Horiguchi, Hitachinaka, Ibaraki, 312-0034, Japan \\ E-mail: tomohiro.naruse.az@hitachi.com \\ ***Department of Mechanical Engineering, Osaka University, \\ 2-1, Yamadaoka, Suita, Osaka, 565-0871, Japan
}

\begin{abstract}
The equivalent stiffness of clamped plates should be prescribed not only to evaluate the strength of bolted joints but also to evaluate deformation and vibration characteristics of practical structures with many bolted joints. The axial stiffness and bending stiffness of clamped plates were evaluated by using Finite Element (FE) analyses while taking the contact condition on bearing surfaces and between the plates into account. We constructed the FE models for bolted joints tightened with M8, 10, 12 and 16 bolts and plate thicknesses of 3.2, 4.5, 6.0 and $9.0 \mathrm{~mm}$, and the axial and bending compliances were precisely evaluated. These compliances of clamped plates were compared with those from VDI 2230 codes (German Engineering Society), in which the equivalent cylindrical and conical compressive stress fields in the plate had been assumed. The compliances of the clamped plates for the preload states were estimated between the predictions as the cylindrical and the conical fields. Meanwhile, the axial and bending compliances of FE analysis can basically be expressed by the VDI 2230 (2003) with the conical field. However, the VDI 2230 (2003) gives the slightly large axial stiffness, and thus the internal force borne to the bolt is predicted in a little bit dangerous failure. Also, it cannot follow the case of clamped plates with different thickness due to assumption of its model.
\end{abstract}

Key words: Bolted Joints, Finite Element Method, Stress Analysis, Axial Stiffness, Bending Stiffness

\section{Introduction}

Strength design guidelines of bolted joints had previously evaluated static collapse, fatigue failure, and slip between fastened plates using stress calculation methods based on material mechanics ${ }^{(1)(6)}$. In these guidelines, it had been assumed that the bolt and the clamped plates had been rigidly tightened with the preload and had been deformed as one body under working loads such as external forces and external moments. Under these deformation conditions, the strength of bolted joints is estimated by using a "joint diagram," which is determined by the stiffness of the bolt and that of the clamped plates. The external load is divided into an additional load on the bolt and one on the clamped plates, depending on the stiffness of the bolt and that of the clamped plates. It is therefore necessary to determine the stiffness of the clamped plates under external forces and external moments.

\footnotetext{
*Received 29 Sep., 2010 (No. T2-09-0118) Japanese Original : Trans. Jpn. Soc. Mech. Eng., Vol. 75, No. 757, A (2009), pp.1230-1238 (Received 12 Feb., 2009) [DOl: 10.1299/jmmp.4.1791]
}

Copyright $\odot 2010$ by JSME 
In order to evaluate the deformation or the vibration characteristic of industrial product structures jointed with a large number of bolts by using finite element (FE) analyses, what is needed is a simplified bolted-joints analysis model with a small number of elements ${ }^{(7) \sim(10)}$. We previously developed a simplified bolted-joints model and a strength evaluation method for this purpose, in which a bolted joint is modelled by an equivalent beam element with total stiffness comprising the stiffness of the bolts and that of the clamped plates ${ }^{(11),(12)}$. In our model, the stiffness of the equivalent beam element is calculated with the axial, bending, and torsional stiffnesses of the bolt and the clamped plates.

It is therefore necessary to precisely estimate not only the axial stiffness of the clamped plates given in the design guidelines but also their bending and torsional stiffnesses in the simplified bolted-joints model in evaluating the strength of bolted joints.

The axial stiffness of the clamped plates had been experimentally (13), (14) and analytically ${ }^{(15) \sim(17)}$ investigated. It was known that there had been significant differences in the axial stiffness equations obtained from experimental results ${ }^{(1)}$. On the other hand, equations obtained via analytical solutions appeared to produce higher stiffness values than those in actual bolted joints, because those solutions had assumed that bolted-joint deformation resulted from the elastic deformation of a cylinder and had made allowance for tension stress between the clamped plates without regarding the contact separation between the plates. In VDI 2230 Blatt1 (2003) code ${ }^{(6)}$, which appears to be the most systematic method in the guidelines, an axial stiffness equation was determined that had been approximated from FE results obtained by Lori et al ${ }^{(18)}$. Lori's FE models had constrained the surface between the clamped plates and had not taken the contact conditions between the plates into consideration. Thus, the axial stiffness equation may have given a higher stiffness value than that in actual bolted joint. Moreover, this axial stiffness was calculated under only clamping force and its value may be different from that calculated under external axial force. On the other hand, no studies appear to have been made in which bending and torsional stiffnesses values were estimated very precisely, excepting some studies with simple but rather imprecise equations based on material mechanics that have been published.

A number of studies on bolted joints had been conducted by many researchers using FE analysis taking the contact condition into consideration. Most of them focused on thread surface contact conditions such as the root stress ${ }^{(19),(20)}$ and nut loosening behaviour according to the thread surface friction ${ }^{(21)}$. However, little consideration was given to the stiffness of the clamped plates, which is most important for evaluating bolted-joint characteristics. In our work, we evaluated the axial stiffness and bending stiffness of clamped plates using FE analyses while taking the contact conditions on bearing surfaces and between plates into account. An FE model was used to characterize the external axial force and bending moment.

\section{Compliance of clamped plates}

Figure 1 shows a schematic depiction of a bolted joint. In the case when two plates are joined with sufficient clamping force and the contact conditions between the two plates are maintained under external force, the subjected external load is transmitted from one plate to the other through the clamp solid shown in the figure. The elastic stiffness of the clamped plates, therefore, is expressed as the elastic stiffness of the clamp solid. According to VDI 2230 (1977) $^{(4)}$ and VDI 2230 Blatt $1(2003)^{(6)}$, the compliances of the clamp solid are calculated as shown below.

In VDI 2230 (1977) ${ }^{(4)}$, the clamp solid is modelled as an equivalent cylinder with an outer diameter equal to the equivalent diameter $D_{e q}$ and an inner diameter equal to the hole diameter $D_{i}$, as shown in the dashed-dotted line of Fig. 2. The equivalent diameter $D_{e q}$ and the equivalent area $A_{e q}$ are defined in Eqns. (1) and (2), respectively. The axial compliance 
of the clamped plates $\delta_{c}$ is obtained from Eqn. (3)

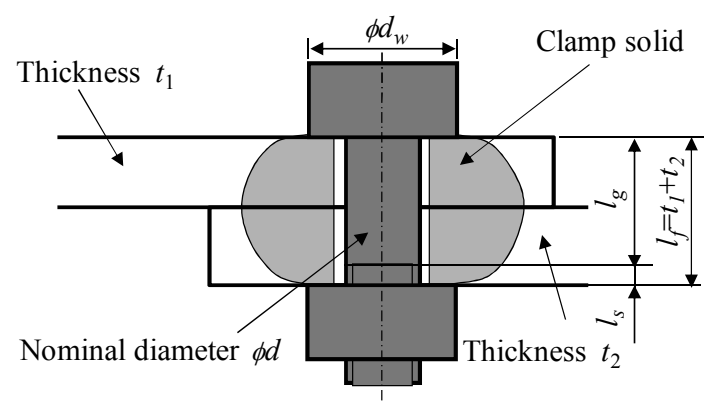

Fig. 1 Structure and model of bolt joints

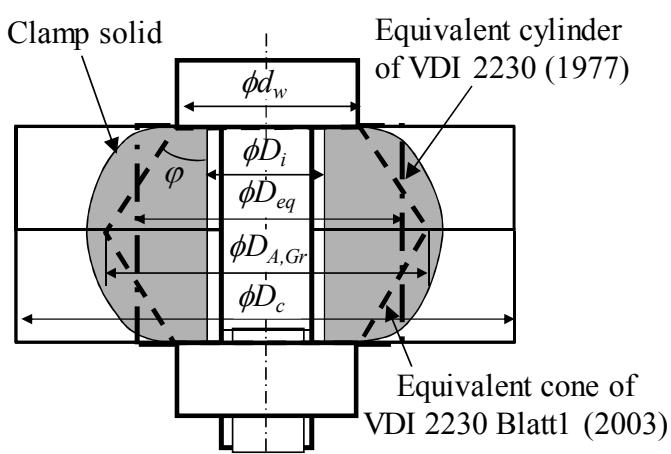

Fig. 2 Equivalent cylinder and equivalent cone

$$
\begin{aligned}
& D_{e q}=d_{w}+\frac{l_{f}}{10} \quad\left(\text { if, } 3 d_{w}<D_{c}, l_{f} \leq 8 d\right) \\
& A_{e q}=\frac{\pi}{4}\left(D_{e q}^{2}-D_{i}^{2}\right) \\
& \delta_{c}=\frac{l_{f}}{E_{c} A_{e q}}
\end{aligned}
$$

In these equations, $d_{w}$ is the outer diameter of the head-bearing surface, $l_{f}$ is the clamping length obtained as a sum of the thicknesses of the clamped plates, $E_{c}$ is Young's modulus of the clamped plates, and $D_{c}$ is the outer diameter of the plates. Equation (1) is applied when the clamped plates are large enough that $D_{c}>3 d_{w}$.

The bending stiffness is not mentioned in VDI $2230(1977)^{(4)}$. Here, we estimated the bending compliance $\beta_{c}$ as that of an equivalent cylinder with an outer diameter equal to the equivalent diameter $D_{e q}$ and an inner diameter equal to the hole diameter $D_{i}$, i.e., in the same manner as in the axial compliance definition.

$$
\beta_{c}=\frac{l_{f}}{E_{c} I_{e q}}=\frac{64 l_{f}}{\pi E_{b}\left(D_{e q}^{4}-D_{i}^{4}\right)}
$$

In VDI $2230(2003)^{(6)}$, the clamp solid is assumed to be an equivalent cone as shown by the dashed lines in Fig. 2. For a through bolted joint, the helix angle of the equivalent cone $\varphi$ is obtained as follows:

$$
\tan \varphi=0.362+0.032 \ln \left(\frac{l_{f}}{2 d_{w}}\right)+0.153 \ln \left(\frac{D_{c}}{d_{w}}\right)
$$

The maximum diameter of equivalent cone $D_{A, G r}$ is calculated as follows:

$$
D_{A, G r}=d_{w}+w l_{f} \tan \varphi
$$

in which $w$ is called the joint coefficient and $w=1$ for a through bolted joint. When the clamped plates are large enough that $D_{c}>D_{A, G r}$, the axial compliance of the clamped plates 
$\delta_{c}$ is obtained by Eqn. (7).

$$
\delta_{c}=\frac{2 \ln \left[\frac{\left(d_{w}+D_{i}\right)\left(d_{w}+w l_{f} \tan \varphi-D_{i}\right)}{\left(d_{w}-D_{i}\right)\left(d_{w}+w l_{f} \tan \varphi+D_{i}\right)}\right]}{w E_{c} \pi D_{i} \tan \varphi}
$$

The bending stiffness is calculated from the second moment of the equivalent cone area as follows:

$$
I_{\text {Bers }}=\frac{3 \pi}{64} \frac{\left(D_{c}-d_{w}\right) d_{w}^{3} D_{c}^{3}}{D_{c}^{3}-d_{w}^{3}}-\frac{\pi}{64} D_{i}^{3} .
$$

Thus the bending compliance of the clamped plates, $\beta_{c}$, is defined in the following equation:

$$
\beta_{c}=\frac{l_{f}}{E_{c} I_{\text {Bers }}} .
$$

We compare these elastic axial and bending compliances of VDI 2230 (1977) and VDI 2230 (2003) with the analysis results obtained with the FE model in the following section.

\section{Analysis model}

\subsection{Analysis model}

Figure 3 shows the FE model of Lori et al ${ }^{(18)}$, from which an equation of the axial compliance in VDI 2230 Blatt1 (2003) ${ }^{(6)}$ had been approximated. The helix angle of the equivalent cone, $\varphi$ shown in Eqn. (5) is obtained from the relation between the tension stress $\sigma_{z S}$ and the axial deformation of the plate at the average diameter of the head-bearing surface, $\Delta l_{K}$ by using multiple regression analysis while varying the parameters of Fig. 3 . Their FE models had constrained the plate's lower surface. Thus, the axial stiffness equation may produce a higher stiffness value than that of an actual bolted joint, and the FE models had been subjected to axial tension load of the bolt. Therefore, the axial compression stiffness of the plate under the clamping force was estimated. However, the axial and bending stiffnesses of plates under external axial load and bending moments had not been considered.

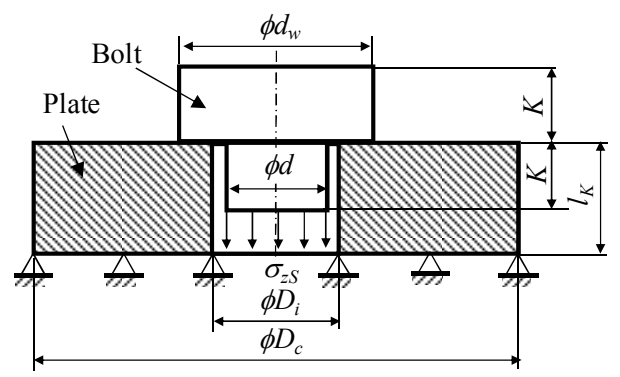

Fig. 3 Lori's model of finite element analysis ${ }^{(18)}$

In this study, we estimated the stiffness of clamped plates under clamping force, axial external force, and bending moment by analysing a 3D FE model that took the contact conditions between clamped plates into consideration. Figure 4 shows the analytical model and its material properties. Figure 5 shows a 3D FE model of Fig. 4. The FE model consisted of two steel plates clamped by a steel bolt. The clamped plates and bolt had the elastic material properties of steel. Contact elements were placed on the upper and lower bearing surfaces and the surface between the plates, and the friction coefficient of these contact surfaces was $\mu=0.15$. The plate thicknesses $t_{1}$ and $t_{2}$ were $3.2,4.5,6.0$, or $9.0 \mathrm{~mm}$ (a thickness of $3.2 \mathrm{~mm}$ being expressed as $t 3.2$ ), and $t_{1}$ and $t_{2}$ were varied with all combinations. The bolt section on the surface between the clamped plates was fixed to the axial direction ( $z$ direction) and the cross point of the bolt axis and the surface between the 
plates was constrained to the $x$ direction. We used a half model because of the symmetry of the bending moment, and the symmetric sections of the bolt and the clamped plates were fixed to the $y$ direction. The diameter of the clamped plates, $D_{c}$, was equal to $5 d_{w}$, a value large enough so as not to affect the contact pressure distribution between the clamped plates. Analyses were carried out with ANSYS ${ }^{\circledR}$ ver. 11 .

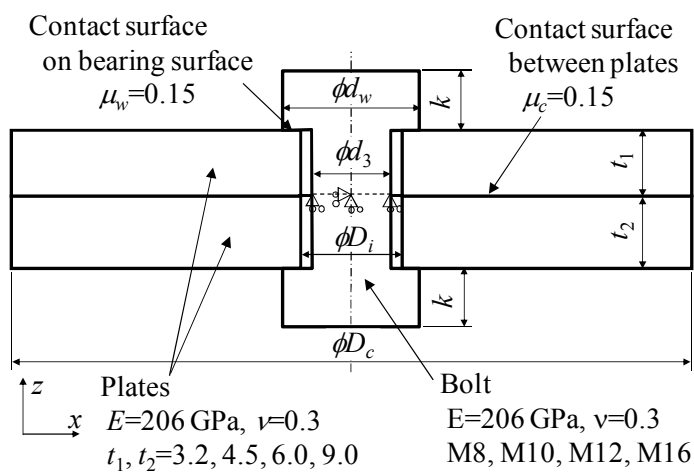

Fig. 4 Dimensions and material properties of analysis model [mm]

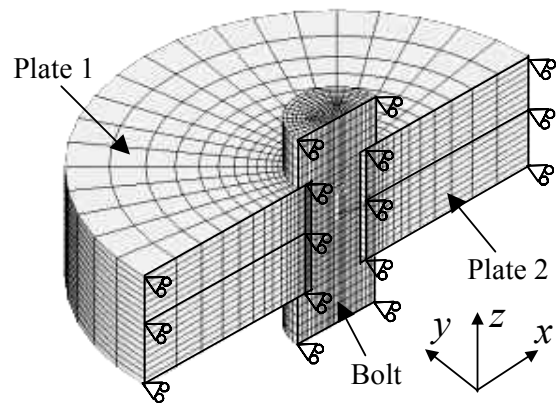

Fig. 5 3D finite element model of bolted joint

Table 1 shows some of the dimensions in Fig. 4, the clamping force (preload) $F_{f}$, the thread stress under the preload $\sigma_{z S}$ and the average pressure on the bearing surface under the preload $p_{w}$. Nominal bolt diameter was from M8 to M16. These bolts were assumed to be full thread bolts, and the bolt model of Fig. 4 was assumed to be cylinders with the minor diameter of the bolt thread. The hole diameter was defined as Class 2 value in JIS B1001. The outer diameter of the bearing surface, $d_{w}$ was defined as being equal to that of a hexagon head with the washer stipulated in JIS B1180. The nut and bolt-head height was equal to that of the hexagon head bolt specified in JIS 1080, though the nut height is higher than that of the bolt head in the actual bolt. The preloads were given so as to be $70 \%$ of the yield pretension defined in JIS 1083. Pretension elements of ANSYS ${ }^{\circledR}$ were applied to the bolt section to give the preload. The thread stresses were $220 \mathrm{MPa}$. On the other hand, the average pressure on the bearing surface was $166 \mathrm{MPa}$.

Table 1 Dimensions of analysis model, preload, preload stress on thread section, and pressure on bearing surface

\begin{tabular}{|cc|c|c|c|c|}
\hline & & M8 & M10 & M12 & M16 \\
\hline$d_{3}$ & {$[\mathrm{~mm}]$} & 6.647 & 8.376 & 10.106 & 13.835 \\
\hline$D_{i}$ & {$[\mathrm{~mm}]$} & 9.0 & 11.0 & 13.5 & 17.5 \\
\hline$d_{w}$ & {$[\mathrm{~mm}]$} & 11.63 & 14.63 & 16.63 & 22.00 \\
\hline$k$ & {$[\mathrm{~mm}]$} & 5.3 & 6.4 & 7.5 & 10.0 \\
\hline$F_{f}$ & {$[\mathrm{~N}]$} & 7,670 & 12,130 & 17,610 & 32,620 \\
\hline$\sigma_{z S}$ & {$[\mathrm{MPa}]$} & 221 & 220 & 220 & 217 \\
\hline$p_{w}$ & {$[\mathrm{MPa}]$} & 180 & 166 & 238 & 234 \\
\hline \multicolumn{2}{|c|}{$d_{w}{ }^{3} / k^{3}$} & 10.57 & 11.95 & 10.902 & 10.648 \\
\hline
\end{tabular}




\subsection{Load conditions}

The axial force and bending moment were supplied to the analysis models shown in Figs. 4 and 5, and the axial and bending stiffness values of the clamped plates were estimated. The apparent stiffness of the clamped plates depends on the external load point ${ }^{(22)}$. Hanau et al. had reported the effect of the load point by using 2D boundary element analysis ${ }^{(22)}$, varying the radial distance from the bearing surface and the height from the plate surface. Their results indicated that the entire external load contributes to deformation on the bearing surface of the clamped plates when the load was applied within the bearing surface. In this work, we aimed to estimate the ideal stiffness of the clamped plates in such cases.

Figure 6 shows the load conditions of the axial force. Negative pressure, which corresponds to the axial load $W_{a}$, was applied to the bearing surfaces of the upper and lower clamped plates. The maximum axial force was loaded around 1.4 times the preload $F_{f}$. Figure 7 shows the load condition of the bending moment. When a bending moment comprising a non-axisymmetric load was applied to an axisymmetric model (such as a cylinder) with axisymmetric elements, it is expanded into the first order Fourier series. Consequently, the result obtained is the first order Fourier integral of the axisymmetric results ${ }^{(23)}$. In this study, 3D solid models were used because nonlinear analyses including contact conditions were carried out. The bending moment was modelled as pressure $p$ of a first order Fourier function, i.e., a cosine function of the angle $\theta, p=100 \cos \theta$ as shown in Fig. 7. The minimum pressure of $-100 \mathrm{MPa}$ was about half of the bearing pressure under the preload, $p_{w}$ shown in Table 1. This moment, therefore, may not open the contact surface between the clamped plates on the tension side and may give enough bending deformation.

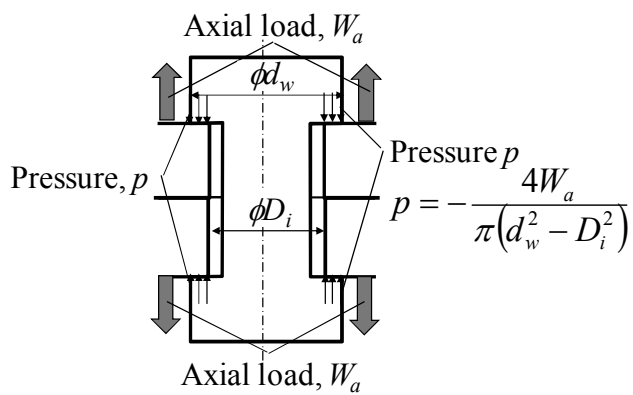

Fig. 6 Axial load condition of analysis model

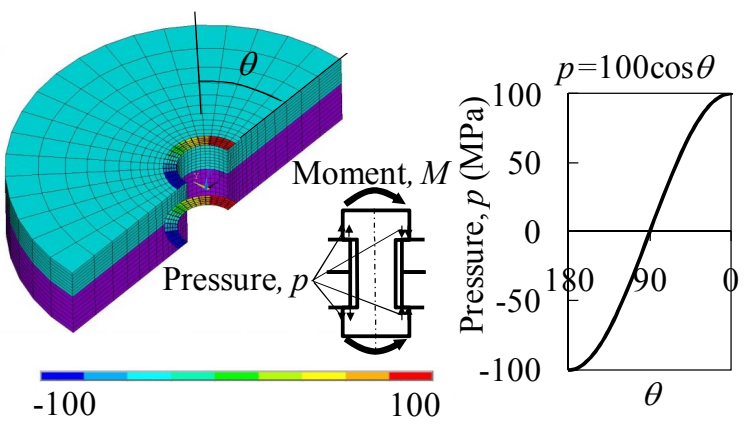

Fig. 7 Bending moment condition of analysis model

\section{Analysis results}

\subsection{Axial compliance}

The axial compliances of the clamped plates under the preload and under the axial external force were calculated for the analysis models in Figs. 4 and 5 under the load condition in Fig. 6, and then they were compared with the values obtained with Eqns. (3) and (7). The compliances were calculated by dividing the compression deformation of the plates by the compression force of the clamped plates. 
First, the compression force was determined. Figure 8 shows the contact pressure between the plates for the result of $t 6.0$ and $t 9.0$ plates fastened by an M10 bolt under the axial force from $W_{a}=0$, i.e. preload, to $1.38 F_{f}$. Figure 9 shows the stress contour of the plates in the $z$ direction, i.e. in the axial direction. Figure 8 shows that the contact pressure reaches a maximum value under the preload and decreases as the axial force is increased. When the axial force is as low as $W_{a}<0.794 F_{f}$, the outer diameter of the pressured area is almost equal to the maximum diameter of the equivalent cone, $D_{A, G r}$ shown in Eqn. (6). The contact pressure between the clamped plates became zero between $W_{a}=1.08 F_{f}$ and $W_{a}=$ $1.38 F_{f}$, because the bolt is elongated and the contact surfaces between plates are separated. From Fig. 9, it can be found that the compressed solid is distributed in the same way as in the equivalent cone, the equivalent cone is maintained when $W_{a}<0.794 F_{f}$, and the cone became small when $W_{a}=1.08 F_{f}$. The compression force $F_{p}$ can be calculated by integrating the contact pressure between the plates shown in Fig. 8 within the entire contact surface between the plates.

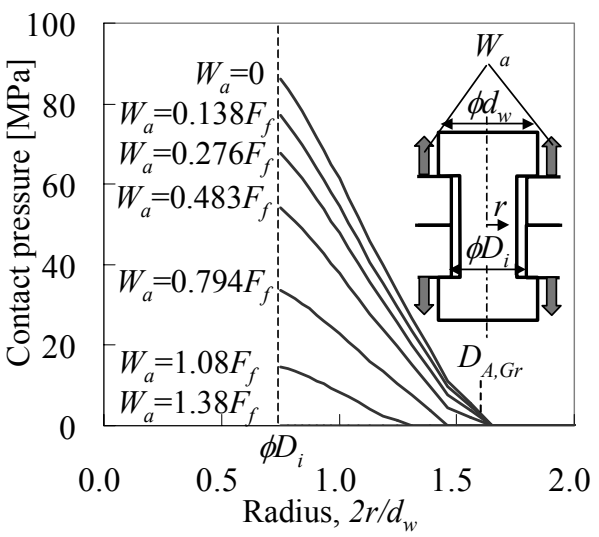

Fig. 8 Contact pressure on surface between two plates under axial load (M10, t6.0, t9.0)

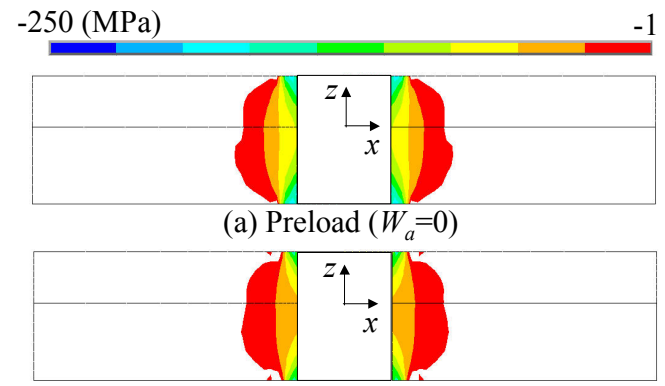

(b) Axial load $\left(W_{a}=0.483 F_{f}\right)$

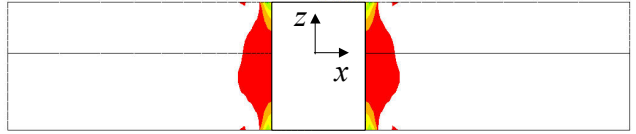

(c) Axial load $\left(W_{a}=1.08 F_{f}\right)$

Fig. $9 \mathrm{z}$ direction stress distribution under preload and axial load (M10, t6.0, t9.0)

Next, the compression deformation of the clamped plates is taken into consideration. Figure 10 shows the contact pressure distribution on the upper bearing surface with $t 6.0$ and t9.0 plates fastened by an M10 bolt. Figure 11 shows the displacement distribution on the upper and lower bearing surfaces with the same model. Figure 10 shows that the contact pressure on the bearing surface reaches a maximum value under the preload and decreases as the axial force is increased, as in Fig. 8. The contact pressure on the bearing surface is maintained from the inner to the outer diameter of the bearing surface, when $W_{a}<0.794 F_{f}$. However, the contact pressure at the outer diameter becomes zero when $W_{a}=1.08 F_{f}$. The centre of contact pressure, $d_{c}$, is almost constant at $0.87 d_{w}$ when $W_{a}<0.794 F_{f}$. 
Comparing the contact pressure between the plates shown in Fig. 8 and that on the bearing surface shown in Fig. 10, both pressures decrease with axial load when the clamped plates are not separated, i.e. $W_{a}<0.794 F_{f}$. When the axial load increases more, i.e. $W_{a}>$ $0.794 F_{f}$, the contact pressure between the plates shown in Fig. 8 becomes zero under $W_{a}=$ $1.38 F_{f}$, though the contact pressure on the bearing surface does not change much from $W_{a}=$ $1.08 F_{f}$ to $W_{a}=1.38 F_{f}$. Lori's analysis model in Fig. 3 had given an axial stiffness of a clamped plate under preload in which the contact pressure on the bearing surface and that between the plates (i.e., equivalent to the reaction force on the lower surface of the plate) were balanced. This study gives an axial stiffness under axial force as well as preload. This axial stiffness, therefore, differs from that of Lori's analysis model, because the contact condition between the plates was taken into consideration under the load condition in which the clamped plates were subjected to not only preload but also axial load.

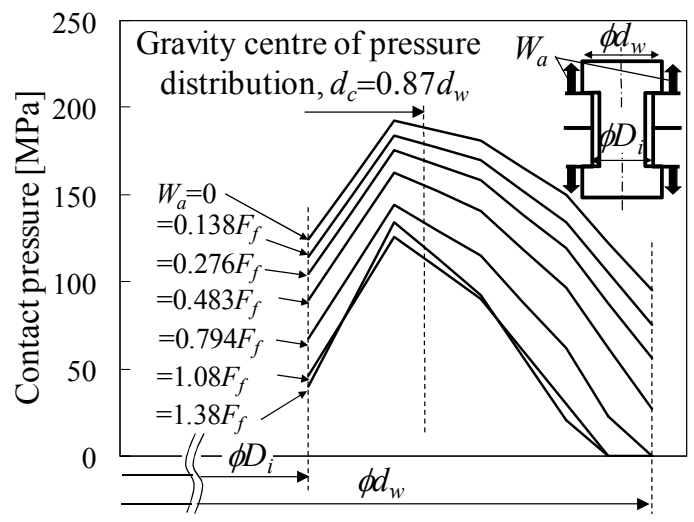

Fig. 10 Contact pressure on upper bearing surface under axial load (M10,t6.0, $t 9.0)$

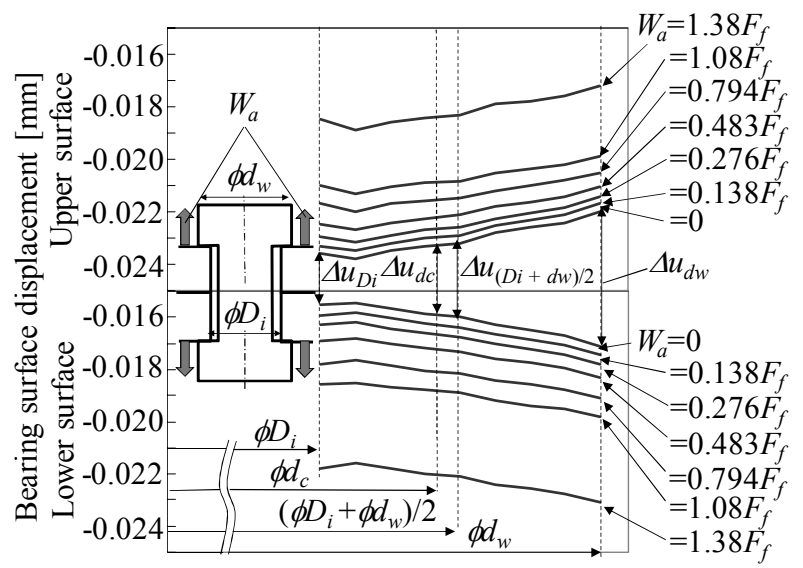

Fig. 11 Displacement on bearing surfaces under axial load (M10,t6.0, t9.0)

The compression deformation of the plates can be calculated by subtracting the displacement of the lower bearing surface from that of the upper bearing surface. Neither the displacement of the hole diameter $D_{i}$ nor the outer diameter of the bearing surface $d_{w}$ are suitable for calculating the compression deformation of the plates because these values are affected by the head deformation. We calculated the displacement difference between the upper and lower bearing surfaces on the hole diameter $D_{i}$ and the outer diameter of the bearing surface $d_{w}$ (referred to as $\Delta u_{D i}$ and $\Delta u_{d w}$ in Fig. 11) and then averaged them (referred to as Ave. $\left.\left(\Delta u_{D i}, \Delta u_{d w}\right)\right)$. We also calculated the displacement difference on the average diameter of $D_{i}$ and $d_{w}$ (referred to as $\Delta u_{(D i+d w) / 2}$ in the figure). In addition to these, the displace difference on the diameter of the centre of pressure, $d_{c}$ was calculated (referred to as $\Delta u_{d c}$ in the figure).

When the axial force was varied, the compression deformations Ave. $\left(\Delta u_{D i}, \Delta u_{d w}\right)$, 
$\Delta u_{(D i+d w) / 2}$ and $\Delta u_{d c}$ changed. Figure 12 shows the relation between the compression force $F_{p}$ and these compression deformations. The compression force and the compression deformation decrease with the axial load $W_{a}$. From Fig. $12, \Delta u_{(D i+d w) / 2}$ and $\Delta u_{d c}$ are almost identical, but Ave. $\left(\Delta u_{D i}, \Delta u_{d w}\right)$ differs from them.

The axial compliance under preload and that under axial force can be estimated from Fig. 12. The axial compliance $\delta_{c}$ under the preload can be calculated by dividing the compression deformation Ave. $\left(\Delta u_{D i}, \Delta u_{d w}\right)$ or $\Delta u_{(D i+d w) / 2}$ by the compression force $F_{p}$ under $W_{a}=0$. The axial compliance obtained under the preload from Ave. $\left(\Delta u_{D i}, \Delta u_{d w}\right)$ was $0.534 \times 10^{-6}[\mathrm{~mm} / \mathrm{N}]$, and that obtained from $\Delta u_{(D i+d w) / 2}$ was $0.593 \times 10^{-6}[\mathrm{~mm} / \mathrm{N}]$, which was about $10 \%$ greater than the former. This indicates that the axial compliance under the preload differs depending on the measurement point of the compression deformation because of the nonlinear deformation on the bearing surface.

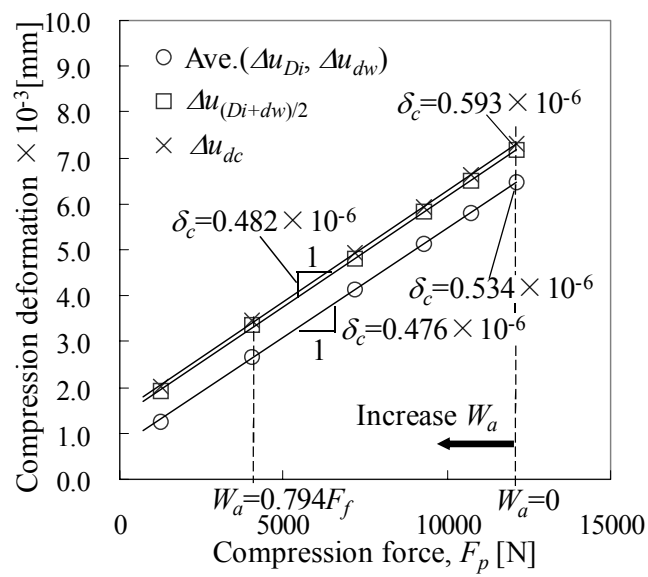

Fig. 12 Relation between compression force and compression deformation of plate (M10, t6.0, t9.0)

On the other hand, the axial compliance $\delta_{c}$ under the axial load can be calculated from the slope of Fig. 12. In this figure, the approximation lines obtained by the charts in $W_{a}<$ $0.794 F_{f}$ are shown as well. The slopes of Ave. $\left(\Delta u_{D i}, \Delta u_{d w}\right)$ and $\Delta u_{(D i+d w) / 2}$ are constant when $W_{a}<0.794 F_{f}$. This indicates that the estimation of the outer diameter of an equivalent cone in VDI 2230 (2003) is valid when the axial load $W_{a}$ was lower than $80 \%$ of the preload. When the axial load is increased to $100 \%$ of the preload, the slopes in Fig. 12 are almost constant, although the compressed solid became small as shown in Fig. 8. The difference between the axial compliances obtained by the slope of Ave. $\left(\Delta u_{D i}, \Delta u_{d w}\right)$ and those obtained by the slope of $\Delta u_{(D i+d w) / 2}$ is less than $2 \%$. Thus, we are able to obtain unique axial compliance.

Next, we estimated the axial compliance under the preload and that under the axial load by using the compression deformation Ave.( $\left(\Delta u_{D i}, \Delta u_{d w}\right)$ for other combinations of clamped plate thickness and bolt nominal diameter.

Figures $13 \sim 16$ show the relations between the axial compliance $\delta_{c}$ and the clamping length $t_{1}+t_{2}$ with M8,10,12, and 16 bolts compared with Eqn. (3) of VDI 2230 (1977) and Eqn. (7) of VDI 2230 (2003). The analysis results are shown as the compliances under the preload and under the axial load. The axial compliance under the preload is larger than that under the axial load. The axial compliance under the preload is placed between the compliance of VDI 2230 (1977), which assumed an equivalent cylinder, and that of VDI 2230 (2003), which assumed equivalent conical compressive stress fields. The tendency of the relations in Figs. $13 \sim 16$ is divided into two groups, one being M8 and M16 and the other being M10 and M12. The cube of the ratio of the outer diameter of bearing surface $d_{w}$ and the bolt head height $k$ are shown in the last row of Table 1. It is considered that $d_{w}{ }^{3} / k^{3}$ represents the inclination deformation of the bolt head. The bolt head tends to significantly incline under preload, when $d_{w}{ }^{3} / k^{3}$ is large. When $d_{w}{ }^{3} / k^{3}$ is small, as in M8 and M16, the 
axial compliance under the preload is likely to be near the value of VDI 2230 (1977). When $d_{w}{ }^{3} / k^{3}$ is large, as in M10 and M12, the inclination deformation of the bolt under the preload becomes large and the compression deformation is estimated as being small, thus it seems that the axial compliance under the preload is much smaller than the value of VDI 2230 (1977).

The axial compliance under the axial load agrees well with the compliance of VDI 2230 (2003), although in several cases the analysis results are $10 \%$ larger than the compliance of VDI 2230 (2003). VDI 2230 (2003) may give higher stiffness because it had been obtained by evaluating an analysis model that fixed the surface between the plates as shown in Fig. 3 . The compliance of VDI 2230 (2003), therefore, was lower than the result we obtained. If the stiffness of the clamped plates were estimated to be higher than the actual stiffness, (i.e. lower compliance than the actual compliance), the bolt stress would be estimated as lower than the actual stress and this would provide an unsafe result. Thus, VDI 2230 (2003) may turn out to provide an unsafe evaluation.

The compliances of analysis results have two values (about $9 \mathrm{~mm}$ and $12 \mathrm{~mm}$ ) of the clamping length $t_{1}+t_{2}$. These were obtained with $t_{1}=t_{2}=4.5$ and $t_{1}=3.2, t_{2}=6.0$ for $t_{1}+t_{2}=9$, and with $t_{1}=t_{2}=6$ and $t_{1}=3.2, t_{2}=9.0$ for $t_{1}+t_{2}=12$, respectively. This shows that the axial stiffness of plates with different thicknesses is divergent from that of plates with the same thickness. Plates with different thicknesses, thus, give higher compliance values than plates with the same thicknesses. When the thicknesses of the clamped plates are very different, their stiffnesses are lower than those given in VDI 2230 (2003), in which the thicknesses are assumed to be the same. This is because the thin plates show large deformation and provide low stiffness values.

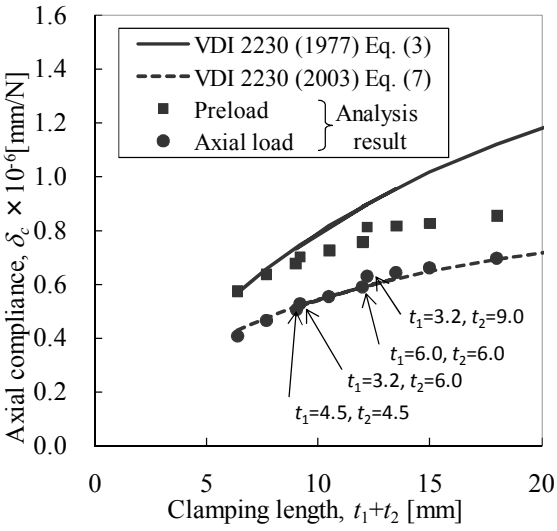

Fig. 13 Axial compliance of clamped plates with M8

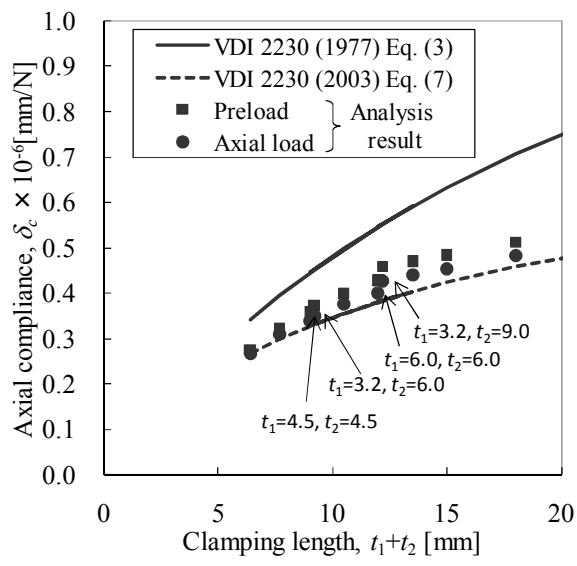

Fig. 15 Axial compliance of clamped plates with M12

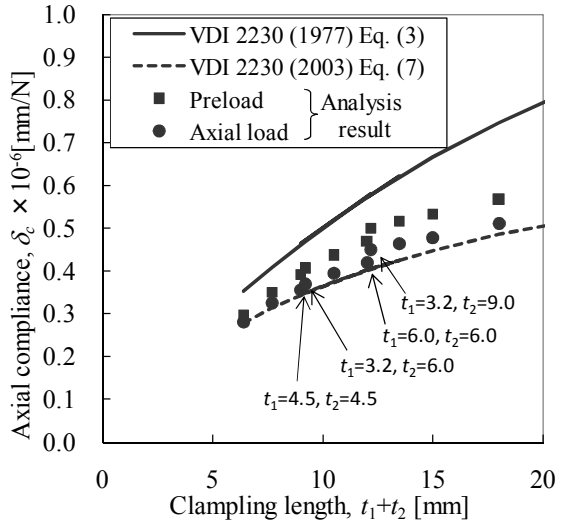

Fig. 14 Axial compliance of clamped plates with M10

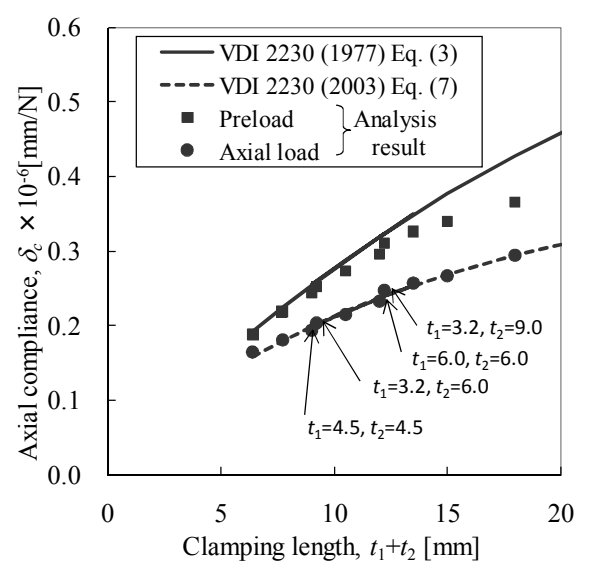

Fig. 16 Axial compliance of clamped plates with M16 


\subsection{Bending compliance}

The bending compliances of the clamped plates under the bending moment were calculated from the analysis models in Figs. 4 and 5 under the bending moment in Fig. 7 after preload. Figure 17 shows the contact pressure between the plates under the bending moment $M$ with $t 6.0$ and $t 9.0$ plates fastened by an M10 bolt. The contact pressure and the contact area increase on the compression side, and they decrease on the tension side under the bending moment. The bending moment of the plates, $M_{p}$ can be calculated by integrating the product of the contact pressure and the $x$ coordinate with the contact area. The bending moment of the plates, $M_{p}$ is lower than the bending moment $M$, because the bolt supports the difference between them.

Figure 18 shows the displacement distribution on the upper and lower bearing surfaces with $t 6.0$ and $t 9.0$ plates fastened by an M10 bolt. Under preload, i.e. $M=0$, the upper bearing surface and the lower bearing surface are parallel. As the bending moment increases, the upper and lower bearing surfaces incline. The bending gradient $\xi$ was defined at the average diameter of the bearing surface, $\left(D_{i}+d_{w}\right) / 2$ by two methods as shown below.

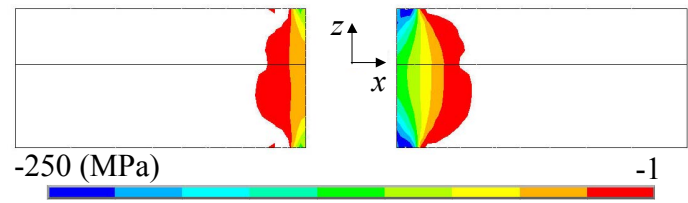

(a) $z$ direction stress

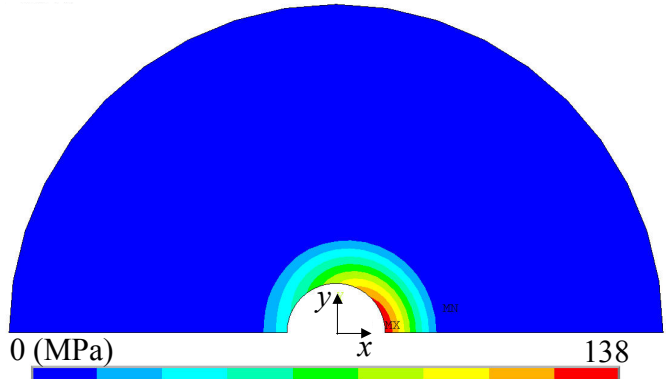

(b) Contact pressure between plates

Fig. $17 z$ direction stress distribution and contact pressure distribution on surface between two plates under bending moment (M10, t6.0, t9.0)

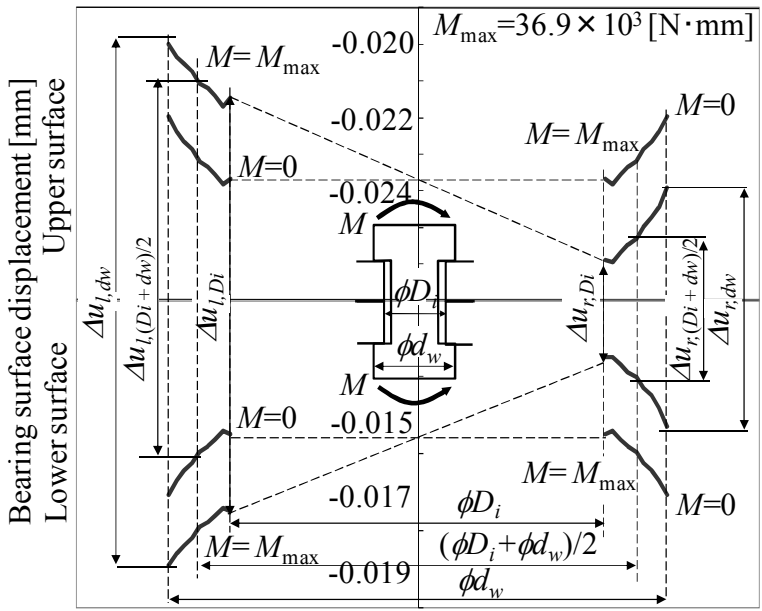

Fig. 18 Displacement on bearing surfaces under bending moment (M10, t6.0, t9.0)

As the first method, the bending gradient $\xi_{1}$ was calculated from the compression deformations of the left and right sides as the averages of the compression deformations at the hole diameter and the outer diameter of the bearing surface, as shown in the following equation: 


$$
\xi_{1}=\frac{\left(\Delta u_{l, D i}+\Delta u_{l, d w}\right)-\left(\Delta u_{r, D i}+\Delta u_{r, d w}\right)}{D_{i}+d_{w}} .
$$

The other method is shown in the next equation; i.e. the bending gradient $\xi_{2}$ was calculated from the compression deformations of the left and right sides at the average diameter of the bearing surface.

$$
\xi_{2}=\frac{2\left(\Delta u_{l,(D i+d w) / 2}-\Delta u_{r,(D i+d w) / 2}\right)}{D_{i}+d_{w}}
$$

Figure 19 shows the relation between the bending moment on the plates $M_{p}$ and the bending gradient $\xi_{1}$ or $\xi_{2}$. The bending compliances $\beta_{c}$ were estimated as the slope of the lines of Fig. 19 and were approximated from four points under the lower bending moments $M_{p}$. The two bending compliances $\beta_{c}$ calculated from $\xi_{1}$ and $\xi_{2}$ are almost identical. When the bending moment $M_{p}$ becomes large, the chart points gradually separate from the approximated lines. The deviation from the approximated line, however, is fairly small. Therefore, we estimated the bending compliance from the bending gradient $\xi_{1}$.

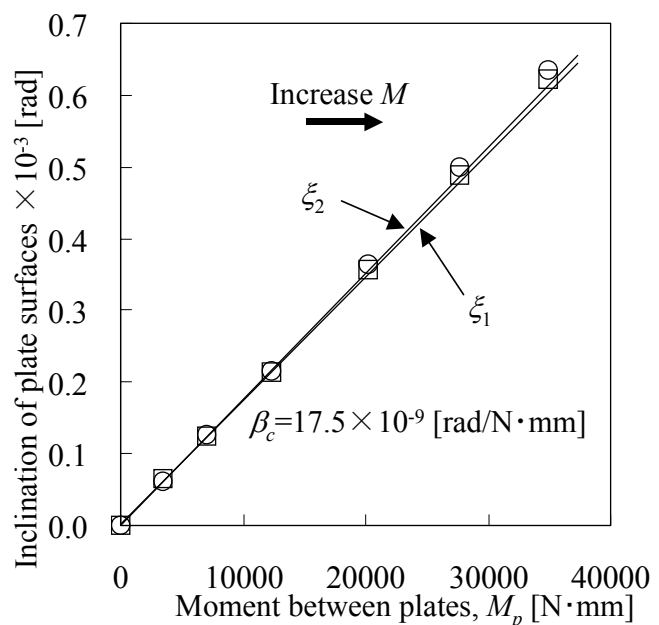

Fig. 19 Relation between moment and inclination of plate surfaces (M10, t6.0, t9.0)

Figures $20 \sim 23$ show the relations between the bending compliance $\beta_{c}$ and the clamping length $t_{1}+t_{2}$ with M8, 10, 12, and 16 bolts compared with Eqn. (4) of VDI 2230 (1977) and Eqn. (9) of VDI 2230 (2003). The analysis results for bending compliance agree well with the compliance of VDI 2230 (2003), although in some cases the results are slightly higher than the compliance values obtained from VDI 2230 (2003). VDI 2230 (2003) may give higher stiffness values because it evaluated an analysis model that fixed the surface between the clamped plates in a manner similar to that for the case of axial compliance.

Higher compliances were obtained for plates with different thicknesses than for those with the same thickness in a similar manner to that used for axial compliance. When the thicknesses of the clamped plates differ greatly, the stiffness of the thin plate affects the total stiffness of the clamped plates, and the stiffness is affected by non-symmetry of the plate thickness. 


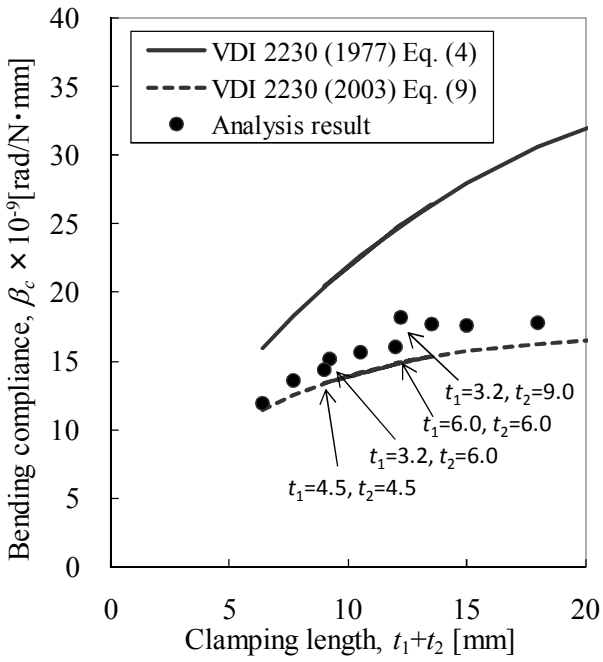

Fig. 20 Bending compliance of clamped plates with M8

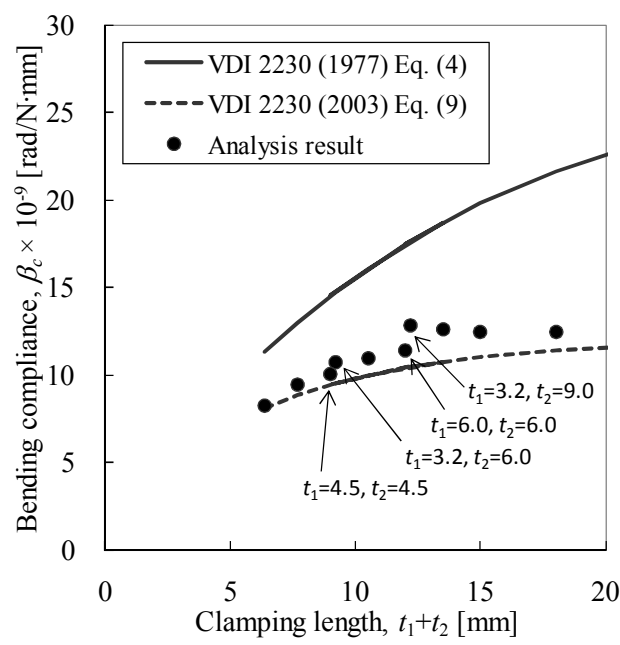

Fig. 22 Bending compliance of clamped plates with M12

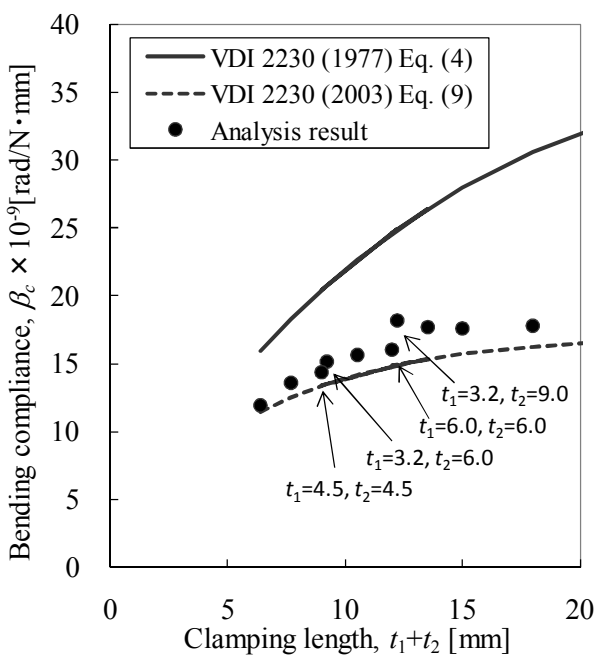

Fig. 21 Bending compliance of clamped plates with M10

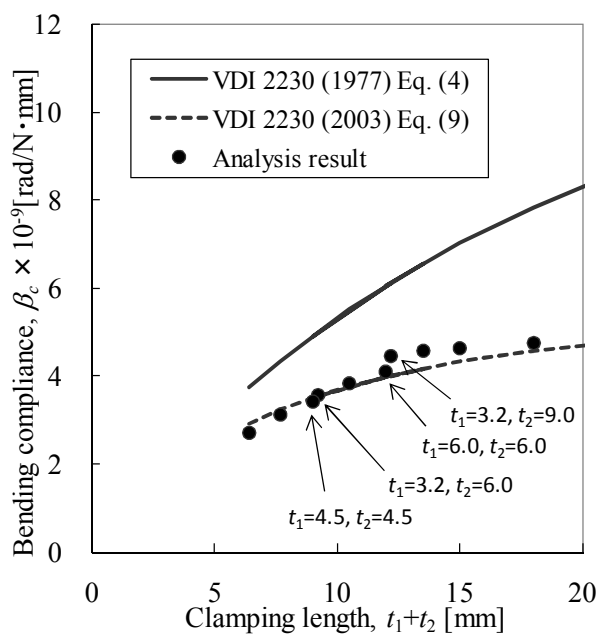

Fig. 23 Bending compliance of clamped plates with M16

\section{Conclusion}

We constructed FE models of bolt-jointed plates with M8, M10, M12, and M16 bolts and plate thicknesses of 3.2, 4.5, 6.0, and $9.0 \mathrm{~mm}$ and subjected them to an axial load and a bending moment. Next, we estimated the axial and bending compliances of the clamped plates from the FE results obtained and compared them with the conventional equations for the equivalent stiffness of the clamped plates. Our procedure in doing so was as follows:

(1) The axial compliance of the clamped plates under preload was placed between the axial compliance of the equivalent cylinder and that of an equivalent cone in the conventional equations.

(2) We estimated the axial compliance of the clamped plates under an axial load by varying the axial load. We found that it was almost constant up to $80 \%$ of the preload and did not show much variation up to $100 \%$ of the preload.

(3) The compliances of the clamped plates were compared with those in the German engineering society code VDI 2230 (2003), in which the equivalent conical compressive stress fields in the plate had been assumed. This code basically expresses the axial and bending compliances of FE analysis. However, it specifies about $10 \%$ larger axial stiffness and thus predicts that internal force borne to the bolt will be slightly smaller than the actual internal force. Therefore, the code gives slightly unsafe estimations. 
(4) When the thicknesses of the clamped plates differed greatly, the compliance of the analysis results was greater than that of VDI 2230 (2003), in which the thicknesses of the clamped plates were assumed to be the same, because the stiffness of the thin plate affected the overall stiffness of the clamped plates.

\section{References}

(1) Yamamoto A., Theory and Design of Bolt Joints (in Japanese), (1977), pp.30, Yokendo .

(2) Yoshimoto, I., Points for Design of Bolt Joints (in Japanese), (1992), pp.177, Japanese Standards Association.

(3) Editorial board of handbook of joint technique, Handbook of Joint Technique (in Japanese), (1994), pp.615, Industrial service center.

(4) Richtlinien VDI 2230, Systematic calculation of high duty bolted joints, (1977), VDI-Verlag [Translated into Japanese by The Japan Research Institute for Screw Threads and Fasteners, (1982), The Japan Research Institute for Screw Threads and Fasteners].

(5) Richtlinien VDI 2230, B1., Systematic calculation of high duty bolted joints, -Joints with one cylindrical bolt-, (1986), VDI-Verlag [Translated into Japanese by Maruyama, K., Gasei, S. and Sawa, T., (1989), The Japan Research Institute for Screw Threads and Fasteners].

(6) Richtlinien VDI 2230, B1., Systematic calculation of high duty bolted joints -Joints with one cylindrical bolt-, (2003), VDI-Verlag [Translated into Japanese by Gasei, S. and Kawai, K., (2006), The Japan Research Institute for Screw Threads and Fasteners].

(7) Kim, J. G., Park, S. S., Choi, S. H. and Kim, B. K., Finite Element Modelling and Experimental Verification of the Structures with Bolted Joints, Transactions of the Korea Society of Mechanical Engineers, Vol. 20, No. 6 (1996), pp.1854-1861.

(8) Hurrell, P. R., Good Practice in Modelling of Pressure Vessel Bolted Joints for Stress and Fatigue Analysis, ASME PVP, Vol. 405 (2000), pp.123-134.

(9) Montgomery, J., Methods for Modeling Bolts in the Bolted Joint, ANSYS 2002 Conference, 2002 April.

(10) Rutman, A. and Kogan, J. B., Software takes the load off joint modeling, Machine Design, Vol. 70, No. 6(1998), pp.79-82.

(11) Naruse, T., Kawasaki, T. and Hattori, T., Simple Modelling and Strength Evaluation Methods for Bolt Joints Using Shell Elements and Beam Elements (1st Report, Modelling Method), Transactions of the Japan Society of Mechanical Engineers, Series A, Vol. 73, No. 728 (2005), pp.522-528 [Translated into English, Journal of Computational Science and Technology, Vol. 3, No. 1 (2009), pp.22-33].

(12) Naruse, T., Kawasaki, T. and Hattori, T., Simple Modelling and Strength Evaluation Methods for Bolt Joints Using Shell Elements and Beam Elements (2nd Report, Strength Evaluation Method), Transactions of the Japan Society of Mechanical Engineers, Series A, Vol. 73, No. 728 (2005), pp. 529-536 [Translated into English, Journal of Computational Science and Technology, Vol. 3, No. 1 (2009), pp.34-45].

(13) Wiegand, H., Illgner, K. H. and Beelich, K. H., Einfluss der Federkonstanten und der Anzugsbedingungen auf die Vorspannung von Schraubenvebindungen, Konstruktion, Vol. 20, No. 4 (1968), pp.130-137.

(14) Thoma, W., Elastische Nachgiebigkeit verspannter Teile einer Sschraubenverbindung, VDI-Z, Vol. 124 (1982), pp.205-214 [Translated into Japanese by Gasei, S., Journal of Japan Research Institute for Screw Threads and Fasteners, Vol. 14, No. 4 (1983), pp.89-100].

(15) Mitsunaga, K., Stress Distribution of Clamped plates of Bolted Joint (in Japanese), Transactions of the Japan Society of Mechanical Engineers, Vol. 31, No. 231 (1965), pp.1750-1757.

(16) For example, Shibahara, M. and Oda, I., Spring Stiffness of Clamped plates of Bolted Joint 
(in Japanese), Transactions of the Japan Society of Mechanical Engineers, Vol. 37, No. 297 (1971), pp. 1033-1040.

(17) For example, Sawa, T. and Shiraishi, H., Simple Calculation Method of Load Factor of Bolted Joint -In the Cases of Cylindrical Flange and Pipe Flange (in Japanese), Transactions of the Japan Society of Mechanical Engineers, Series C, Vol. 48, No. 434 (1982), pp.1598-1606.

(18) Lori, W., und Glaser, H., Berechnung der Plattennachgiebigkeit bei Schraubenverbindungen, Konstruktion, Vol. 42 (1990), pp.271-277 [Translated into Japanese by Gasei, S., Journal of Japan Research Institute for Screw Threads and Fasteners, Vol. 22, No. 12 (1991), pp.393-401].

(19) For example, Tanaka, M., Hongo, K. and Asaba, E., Application of Finite Element Method for Bolted Joint, Transactions of the Japan Society of Mechanical Engineers, Series $C$, Vol. 48, No.434 (1982), pp.1607-1614.

(20) For example, Fukuoka, T., Nomura, M. and Morimoto, Y., Proposition of Helical Thread Modeling with Accurate Geometry and Finite Element Analysis, Transactions of the Japan Society of Mechanical Engineers, Series A, Vol. 72, No. 723 (2006), pp.1639-1645.

(21) For example, Izumi, S., Yokoyama, T., Iwasaki, A. and Sakai, S., Three-dimensional Finite Element Analysis of Tightening and Loosening Mechanism of Bolted Joint, Transactions of the Japan Society of Mechanical Engineers, Series A, Vol. 71, No. 702 (2005), pp.204-212.

(22) Hanau, A., Zum Kraftleitungsverhalten zentrisch verspannter Schraubenverbindungen., Diss. TU Berlin (1994).

(23) ANSYS v.11.0 Documentation, 2.13 Axisymmetric Elements with Nonaxisymmetric Loads, ANSYS. 